\title{
Wireless Power Transfer System with Ideal Transformer Characteristics Determined Solely by Coil Turns Ratio
}

\author{
Kenji Nara $^{* a)} \quad$ Student Member, Naofumi Madoiwa** ${ }^{*}$ Student Member \\ Keiri Maeshiro* Student Member, Yasuyoshi Kaneko* Member \\ (Manuscript received Feb. 4, 2020, revised May 7, 2020) \\ J-STAGE Advance published date : October 1, 2020
}

\begin{abstract}
To manufacture wireless power transfer (WPT) products, not only in-depth knowledge of WPT but also various factors, such as power supply, charging circuit, and battery, must be considered, and the complexity is a barrier to the spread of WPT technology. Therefore, if the voltage is easily stepped up/down by the WPT circuit section, a generalpurpose power supply and charging circuit can be used, leading to simplification of the system. The S/SP method proposed in this study exhibits the same input/output (I/O) characteristic of an ideal transformer, and the I/O ratio of the WPT circuit is determined from the turn ratio of the coils, similar to a general transformer. In a power feeding experiment, the I/O voltage ratio of the WPT circuit matched the turn ratio of the coils. The power transfer efficiency of the S/SP system was lower than that of the S/P system, but the difference was only $1.0 \%$. Through a secondary side misalignment experiment, we confirmed that the $\mathrm{I} / \mathrm{O}$ relation was maintained to an extent. In addition, the S/SP method was clarified to have better displacement characteristics than those of the $\mathrm{S} / \mathrm{P}$ method.
\end{abstract}

Keywords: wireless power transfer, constant voltage output, S/SP system, ideal transformer, circuit design

\section{Introduction}

Wireless power transfer (WPT) is being considered to be a promising technology. It is a convenient charging way in which power is supplied to a portable device such as a smartphone and laptop computer by just placing it on a wireless charger $^{(1)(2)}$. Also, if it can be mounted on desktop devices such as TVs and desktop computers, WPT technology increases installation flexibility of the devices and simplify the complicated power cables behind the desk.

In WPT based on magnetic field coupling, which is called inductive power transfer ${ }^{(3)}$, inductors and capacitors are generally connected in series or parallel to transmitting and receiving coils to improve circuit characteristics ${ }^{(4)(5)}$. The series/series (S/S) WPT system has been widely studied ${ }^{(6)}$ because of its excellent circuit characteristics such as a theoretical maximum efficiency and input power factor $\left(P F_{I N}\right)$ of 1 . However, with a constant voltage (CV) power supply driving, the output of the $\mathrm{S} / \mathrm{S}$ circuit is a constant current (CC). Hence, the output voltage changes due to battery load fluctuations. Therefore, it is necessary to control a converter connected to the charging circuit to prevent the voltage from changing considerably. In addition, it is necessary to obtain a circuit design that does not exceed the rated voltage and

a) Correspondence to: Kenji Nara. E-mail: k.nara.0921@gmail. com

* Graduate School of Science and Engineering, Saitama University

Saitama 338-8570, Japan

** Department of Electrical and Electronic Engineering, Tokyo Institute of Technology

Tokyo 152-8552, Japan current of a power supply, charging circuit, and battery, and the complexity limits the spread of WPT technology. If the voltage is easily stepped up/down by the WPT circuit section, a general-purpose power supply and charging circuit can be used, resulting in a simpler system. Also, in the case that the $\mathrm{I} / \mathrm{O}$ voltage can be determined and fixed in the WPT section, a voltage control or a converter for the voltage control can be omitted, which leads to simplification, miniaturization and high efficiency of the system.

Therefore, in this paper, we present a solution to promote the introduction of WPT technology by proposing WPT circuits that it is easy to convert voltage in WPT section. Here, designability is defined as follows, and WPT circuits are proposed and evaluated based on them.

1) The output of WPT circuit is constant voltage.

2) WPT systems do not require a voltage control.

3) Input/output voltage can be determined arbitrarily.

Our team studied a WPT circuit with the ideal transformer characteristics as a circuit that satisfies the above conditions ${ }^{(7)}$. In that research, we proposed a new S/SP compensation method in which the $\mathrm{I} / \mathrm{O}$ voltage ratio is determined from the self-inductance or turn ratio of the coils alone. Although the S/SP method was dealt with in a few studies ${ }^{(8)-(10)}$, no comparison of transmitting efficiency and experimental comparison of secondary misalignment were made with the $\mathrm{S} / \mathrm{P}$ method ${ }^{(11)(12)}$ whose circuit configuration and circuit characteristics are similar. In this study, these two topologies are compared and evaluated through simulations and experiments. In addition, our previous work is extended to examine the change due to the secondary coil displacement, and the superiority of the S/SP method is clarified.

The remainder of this paper is organized as follows: 


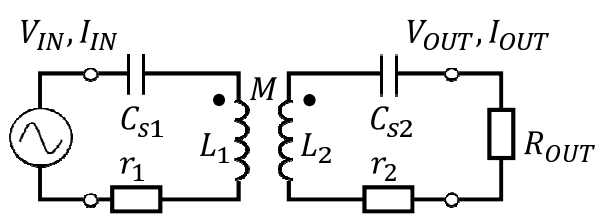

Fig. 1. WPT circuit with S/S method

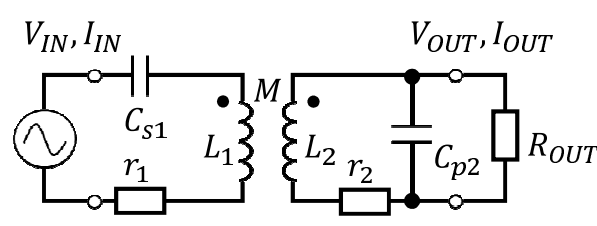

Fig. 2. WPT circuit with S/P method section 2 analyzes the S/S and S/P topology and describes the characteristics of $\mathrm{CC}$ and $\mathrm{CV}$ output. Section 3 presents an analysis of a WPT circuit which takes on the characteristic of an ideal transformer. Section 4 discusses the simulation and experimental results verifying the analysis and compares $\mathrm{S} / \mathrm{SP}$ and S/P topologies. Furthermore, the characteristics of secondary misalignment are clarified by simulation and experiment, comparing both approaches. Finally, section 5 concludes the study and states the direction of our future work.

\section{Conventional WPT System}

Figure 1 shows a simplified S/S WPT circuit. $L_{1}, L_{2}$, and $M$ are the self-inductance and mutual inductance of the primary and secondary side coils, respectively. These follow relationship (1) via the coupling coefficient $k$ :

$$
M=k \sqrt{L_{1} L_{2}} .
$$

\subsection{S/S WPT System with Constant Current Output}

In (2), capacitances $C_{s 1}$ and $C_{s 2}$ on the primary and secondary sides resonate with $L_{1}$ and $L_{2}$ respectively at the resonant frequency $\omega=2 \pi f$ :

$$
C_{s 1}=\frac{1}{\omega^{2} L_{1}}, \quad C_{s 2}=\frac{1}{\omega^{2} L_{2}} .
$$

If the winding resistances $r_{1}$ and $r_{2}$ are sufficiently small with respect to the reactance component that they can be ignored, the I/O characteristic of the circuit is expressed by (3) using the F-matrix. Here, $V_{I N}, V_{O U T}, I_{I N}$ and $I_{O U T}$ represent input voltage, output voltage, input, current and output current, respectively:

$$
\left(\begin{array}{l}
V_{I N} \\
I_{I N}
\end{array}\right)=\left(\begin{array}{cc}
0 & -j \omega M \\
\frac{1}{j \omega M} & 0
\end{array}\right)\left(\begin{array}{l}
V_{\text {OUT }} \\
I_{\text {OUT }}
\end{array}\right)
$$

When the capacitances are determined by (2), the output becomes CC with CV driving, as shown in (3). Therefore, it is difficult to keep maintain output voltage against load fluctuation and to obtain $\mathrm{I} / \mathrm{O}$ voltage and power with the desired value. In practice, a DC-DC converter is used at the output of the WPT circuit to control the voltage and power applied to the load ${ }^{(13)-(16)}$. In contrast, a WPT circuit with CV output eliminates feedback control at the output. This makes it possible to use a general-purpose converter or omit a converter itself, which is advantageous in system simplicity and cost reduction.

2.2 S/P WPT System The S/P method shown in Fig. 2 is a typical WPT circuit topology with CV output during $\mathrm{CV}$ driving. By determining compensation capacitors as shown in (4), a high efficiency, high $P F_{I N}$, and CV output system can be achieved:

$$
C_{s 1}=\frac{1}{\omega^{2}\left(1-k^{2}\right) L_{1}}, \quad C_{p 2}=\frac{1}{\omega^{2} L_{2}} . \cdots \cdots \cdots \cdots
$$

The I/O characteristic of $\mathrm{S} / \mathrm{P}$ circuit is expressed by (5):

$$
\left(\begin{array}{l}
V_{I N} \\
I_{I N}
\end{array}\right)=\left(\begin{array}{cc}
\frac{M}{L_{2}} & 0 \\
0 & \frac{L_{2}}{M}
\end{array}\right)\left(\begin{array}{c}
V_{O U T} \\
I_{O U T}
\end{array}\right)
$$

There is no voltage fluctuation due to the load change because the $\mathrm{S} / \mathrm{P}$ method has a $\mathrm{CV}$ output characteristic as seen from (5). However, since the voltage gain includes the mutual inductance, coils having the same specification cannot be used for products having different distances between power transmission and reception side, which is disadvantageous for mass production.

\subsection{S/S WPT System with Constant Voltage Output}

Contrary to the discussion in section 2.1 , it has been reported that by driving the power supply at offset frequencies with respect to the resonant frequency $f$, the output characteristic is changed, and a CV output is obtained at certain frequencies ${ }^{(7)(17)(18)}$. In those works, it was shown that by driving at the frequency $\omega_{1}=2 \pi f_{1}$ represented by (6), the $\mathrm{I} / \mathrm{O}$ relationship of CV is obtained even in the S/S system:

$$
f_{1}=\frac{1}{\sqrt{1 \pm k}} f
$$

Shifting the power supply frequency is equivalent to shifting the resonant frequency of the compensation capacitors. In other words, when the power supply is driven at $f$ in the $\mathrm{S} / \mathrm{S}$ system, a CV output can be obtained by determining the compensation capacitors as follows:

$$
C_{s 1}=\frac{1}{\omega^{2}(1 \pm k) L_{1}}, \quad C_{s 2}=\frac{1}{\omega^{2}(1 \pm k) L_{2}} . \cdots \cdots
$$

Consequently, the $\mathrm{I} / \mathrm{O}$ characteristic of the circuit is given by:

$$
\left(\begin{array}{l}
V_{I N} \\
I_{I N}
\end{array}\right)=\left(\begin{array}{cc}
\mp \sqrt{\frac{L_{1}}{L_{2}}} & 0 \\
\frac{1}{j \omega M} & \mp \sqrt{\frac{L_{2}}{L_{1}}}
\end{array}\right)\left(\begin{array}{c}
V_{\text {OUT }} \\
I_{\text {OUT }}
\end{array}\right)
$$

Equation (8) shows a CV output under CV driving. Moreover, as the non-diagonal components are not all $0, P F_{I N}$ is not 1 .

\section{WPT Systems with Ideal Transformer Char- acteristics}

3.1 Analysis of Proposed WPT Circuits When $C_{s 1}$ and $C_{s 2}$ are determined using (7), it can be seen that the input impedance of the WPT circuit includes an imaginary component and $P F_{I N}$ is not 1 . Therefore, an impedance $Z_{p}$, in the form of an inductor or capacitor, is connected for power factor compensation such that the non-diagonal component of (8) is 0. As shown in Fig. 3, the I/O characteristics of the circuit are expressed as (9) and (10) by connecting an impedance in parallel on the power supply side or the load side. Here, (9) and (10) correspond to Figs. 3(a) and 3(b), 


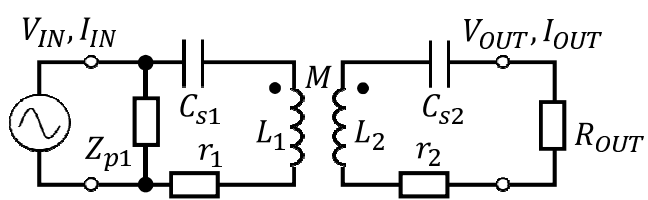

(a) Parallel compensation before $\mathrm{S} / \mathrm{S}$ circuit

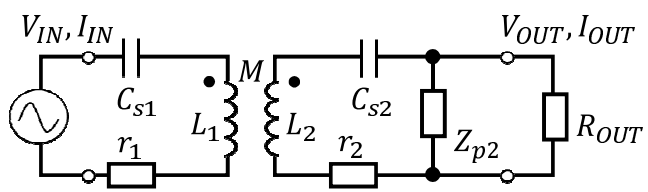

(b) Parallel compensation after $\mathrm{S} / \mathrm{S}$ circuit

Fig. 3. WPT circuit with CV output based on S/S method

respectively:

$$
\begin{aligned}
& \left(\begin{array}{l}
V_{I N} \\
I_{I N}
\end{array}\right)=\left(\begin{array}{cc}
\mp \sqrt{\frac{L_{1}}{L_{2}}} & 0 \\
\frac{1}{j \omega M} \mp \frac{1}{Z_{p 1}} \sqrt{\frac{L_{1}}{L_{2}}} & \mp \sqrt{\frac{L_{2}}{L_{1}}}
\end{array}\right)\left(\begin{array}{l}
V_{\text {OUT }} \\
I_{\text {OUT }}
\end{array}\right), \ldots \ldots \\
& \left(\begin{array}{c}
V_{I N} \\
I_{I N}
\end{array}\right)=\left(\begin{array}{cc}
\mp \sqrt{\frac{L_{1}}{L_{2}}} & 0 \\
\frac{1}{j \omega M} \mp \frac{1}{Z_{p 2}} \sqrt{\frac{L_{2}}{L_{1}}} & \mp \sqrt{\frac{L_{2}}{L_{1}}}
\end{array}\right)\left(\begin{array}{l}
V_{\text {OUT }} \\
I_{\text {OUT }}
\end{array}\right) \ldots \ldots
\end{aligned}
$$

$Z_{p 1}$ and $Z_{p 2}$ are adjusted appropriately so that the nondiagonal component is 0 . When $Z_{p}>0$, an inductor should be connected, and when $Z_{p}<0$, a capacitor should be connected. Table 1 summarizes each compensation method and I/O relationship.

In the circuit shown in Fig. 3, when the compensation circuit conforms to Table 1, the I/O relation of the WPT circuit has the same characteristic as an ideal transformer. Thus, the voltage ratio can be determined solely from the selfinductance. Since it does not include parameters that are determined by the positional relationship of the coils, such as the coupling coefficient (or the mutual inductance), a circuit design using this topology is straightforward. In the previously proposed WPT topologies, the I/O relationship depends on the coupling coefficient, so it is necessary to temporarily create coils, measure the coupling factor, and rewind the coils when performing voltage step up/down in the WPT circuit section. On the other hand, our proposed method does not require such a rewinding (or temporary creation) process. Furthermore, unlike the S/P method, a coil that achieves a certain design requirement can satisfy the same requirement even for products with different gaps between transmitting and receiving coils, simply by changing capacitors.

Here, four WPT topologies have been proposed; however, it is generally more difficult to obtain an inductor with a desired value than a capacitor. Only compensation by capacitors is considered because this study focuses on the ease of design. In addition, when a parallel capacitor is connected to the first compensation circuit, as viewed from the power supply, the fundamental input impedance is larger than the impedance to the harmonics. This is not suitable for a square wave input. Therefore, the following discussion deals with the S/SP WPT system (which is listed in Table 1 as case D).

3.2 Condition of Ideal Transformer Characteristics

A condition that I/O relationship of S/SP system is determined by the turn ratio of coils is mentioned using solenoid type coils in this section. The relationship between the selfinductance of the solenoid coil and the number of turns of the coil is expressed as:

$$
L_{i}=\frac{\mu_{0 i} S_{i}}{l_{i}} N_{i}^{2}
$$

Here, $i$ is 1 or 2 , indicating the transmitting or receiving side, $\mu_{0}$ is the permeability, $S$ is the cross-sectional area of the coil, and $l$ is the coil length. If these parameters are equal for both sides, that is:

$$
\mu_{01}=\mu_{02}, \quad S_{1}=S_{2}, \quad l_{1}=l_{2}
$$

then the $\mathrm{I} / \mathrm{O}$ characteristic of proposed circuit is rewritten as below:

$$
\left(\begin{array}{c}
V_{I N} \\
I_{I N}
\end{array}\right)=\left(\begin{array}{cc}
\frac{N_{1}}{N_{2}} & 0 \\
0 & \frac{N_{2}}{N_{1}}
\end{array}\right)\left(\begin{array}{c}
V_{\text {OUT }} \\
I_{\text {OUT }}
\end{array}\right) .
$$

That is, when the capacitances of S/SP circuit are determined as Table 1 and coils with the same shape and dimensions are used on both the primary and secondary sides, the I/O ratio of the WPT circuit can be determined by the turn ratio of the coils alone, as in a general transformer.

\section{Verification by Experiment and Simulation}

Based on the theory proposed in Section 3, we conducted power feeding experiments and simulations. Solenoid coils as shown in Fig. 4 were created. Litz wire with 22 turns on the primary side and 11 turns on the secondary side was wound around a Mn-Zn ferrite core (NJ Components Co., Ltd., 6H45). Turn rario of the coils was 2.00. The distance from the center of the primary coil to the center of the secondary coil was $25 \mathrm{~mm}$. Every parameter for the coils in this condition is listed in Table 2. We also conducted the same experiments and simulations for the $\mathrm{S} / \mathrm{P}$ system and the $\mathrm{S} / \mathrm{S}$ system with CV output (S/S (CV) system) and compared it with the S/SP system. Figure 5 shows the experimental and simulation circuit structures with the conditions listed

\begin{tabular}{|c|c|c|c|c|c|c|c|}
\hline Case & Parallel impedance & Parallel inductor/capacitor & Series capacitor & \multicolumn{4}{|c|}{$\mathrm{I} / \mathrm{O}$ relation } \\
\hline A & \multirow{2}{*}{$Z_{p}=j \omega L_{p}$} & $L_{p 1}=k L_{1}$ & \multirow{2}{*}{$\begin{array}{l}C_{s 1}=\frac{1}{\omega^{2}(1+k) L_{1}} \\
C_{s 2}=\frac{1}{\omega^{2}(1+k) L_{2}}\end{array}$} & \multirow[t]{2}{*}{$\left(V_{I N}\right)=$} & \multirow{2}{*}{$\left(\begin{array}{c}-\sqrt{\frac{L_{1}}{L_{2}}} \\
0\end{array}\right.$} & \multirow{2}{*}{$\left.\begin{array}{c}0 \\
-\sqrt{\frac{L_{2}}{L_{1}}}\end{array}\right)$} & \multirow{2}{*}{$\mid \begin{array}{l}V_{\text {OUT }} \\
I_{\text {OUT }}\end{array}$} \\
\hline B & & $L_{p 2}=k L_{2}$ & & & & & \\
\hline $\mathrm{C}$ & \multirow{2}{*}{$Z_{p}=\frac{1}{j \omega C_{p}}$} & $C_{p 1}=\frac{1}{\omega^{2} k L_{1}}$ & \multirow{2}{*}{$\begin{array}{l}C_{s 1}=\frac{1}{\omega^{2}(1-k) L_{1}} \\
C_{s 2}=\frac{1}{\omega^{2}(1-k) L}\end{array}$} & \multirow{2}{*}{$\left(\begin{array}{l}V_{I N} \\
I_{I N}\end{array}\right)=$} & \multirow{2}{*}{$=\left(\begin{array}{c}\sqrt{\frac{L_{1}}{L_{2}}} \\
0\end{array}\right.$} & 0 & \multirow{2}{*}{$\left(\begin{array}{l}V_{\text {OUT }} \\
\\
I_{\text {OUT }}\end{array}\right)$} \\
\hline $\mathrm{D}$ & & $C_{p 2}=\frac{1}{\omega^{2} k L 2}$ & & & & $\left.\sqrt{\frac{L_{2}}{L_{1}}}\right)$ & \\
\hline
\end{tabular}
in Table 3. In the transmitting side, DC voltage made by regulated power supply was converted to square $\mathrm{AC}$ voltage by the full-bridge DC/AC converter with MOSFET module

Table 1. Compensation method for ideal transformer characteristic and its I/O relation 


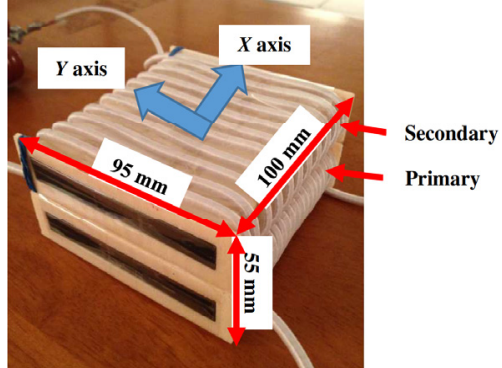

Fig. 4. Solenoid-type coil

Table 2. Coil parameters

\begin{tabular}{cc|cc}
\hline Parameter & Value & Parameter & Value \\
\hline$N_{1}$ [Turn] & 22 & $L_{1}[\mu \mathrm{H}]$ & 85.1 \\
$N_{2}$ [Turn] & 11 & $L_{2}[\mu \mathrm{H}]$ & 21.6 \\
$k$ & 0.455 & $M[\mu \mathrm{H}]$ & 19.5 \\
$Q_{1}$ & 465 & $r_{1}[\Omega]$ & 0.0977 \\
$Q_{2}$ & 446 & $r_{2}[\Omega]$ & 0.0259 \\
$N_{1} / N_{2}$ & 2.00 & $\sqrt{L_{1} / L_{2}}$ & 1.98 \\
\hline
\end{tabular}

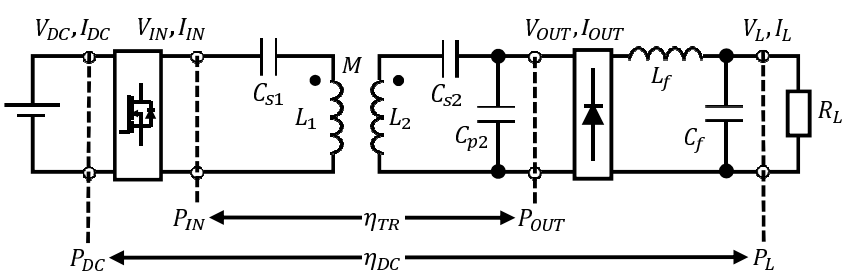

Fig. 5. Experimental/simulation circuit

(SPC Electronics Co., SFM60-11). Behind of WPT circuit, $\mathrm{AC}$ voltage was converted to DC voltage by the full-bridge rectifier with fast recovery diode (STMicroelectronics N. V., STTH12012TV1) and by LC smoothing filter whose values were $60 \mu \mathrm{H}$ on the inductor and $1000 \mu \mathrm{F}$ on the capacitor. In the simulations and experiments, the same DC power supply, DC/AC converter, coil, rectifier, and smoothing filter were used even in each method, but there was no smoothing inductor in the $\mathrm{S} / \mathrm{S}(\mathrm{CV})$ system because the output current of $\mathrm{S} / \mathrm{S}(\mathrm{CV})$ WPT circuit was square wave without $L_{f}$. The power supply frequency was $85.0 \mathrm{kHz}$. Table 3 also shows the theoretical optimum output resistance $R_{\text {OUTopt }}$ and maximum efficiency $\eta_{\max }$. $R_{\text {OUTopt }}$ of each circuit was calculated based on Ref. (7) and Ref. (12). The load resistance $R_{L}$ was determined so that $R_{\text {OUTopt }}$ was equal to the equivalent $\mathrm{AC}$ resistance ${ }^{(19)(20)}$. Hence, $R_{L}$ was determined as:

$$
R_{\text {OUTopt }}=\frac{\pi^{2}}{8} R_{L} \ldots \ldots \ldots \ldots \ldots \ldots \ldots \ldots \ldots
$$

for $\mathrm{S} / \mathrm{SP}$ and $\mathrm{S} / \mathrm{P}$ method and as:

$$
R_{\text {OUTopt }}=\frac{8}{\pi^{2}} R_{L} \ldots \ldots \ldots \ldots \ldots \ldots \ldots \ldots \ldots \ldots
$$

for $\mathrm{S} / \mathrm{S}(\mathrm{CV})$ method. $\eta_{\max }$ of the S/SP method was $98.5 \%$, which was lower than that of $\mathrm{S} / \mathrm{P}$ method, but the difference was only $0.4 \%$, and it can be said that the S/SP is a highly efficient circuit topology.

4.1 Power Feeding in Secondary Alignment The power feeding experiment was performed when the primary and secondary coils were facing each other. Here, the DC input voltage $V_{D C}$ was adjusted so that the load power $P_{L}$ applied to the load resistance was $800 \mathrm{~W}$. Table 4 lists the root
Table 3. Experimental/simulation conditions and theoretical optimal load and maximum efficiency

\begin{tabular}{cccc}
\hline Parameter & S/SP & S/P & S/S (CV) \\
\hline$f[\mathrm{kHz}]$ & 85.0 & 85.0 & 85.0 \\
$R_{L}[\Omega]$ & 5.91 & 21.9 & 9.37 \\
$C_{s 1}[\mu \mathrm{F}]$ & 0.0755 & 0.0526 & 0.0755 \\
$C_{S 2}[\mu \mathrm{F}]$ & 0.299 & - & 0.299 \\
$C_{p 2}[\mu \mathrm{F}]$ & 0.367 & 0.161 & - \\
$R_{\text {OUTopt }}[\Omega]$ & 7.29 & 27.0 & 7.59 \\
$\eta_{\max }[\%]$ & 98.5 & 98.9 & 98.6 \\
\hline & & &
\end{tabular}

Table 4. Experimental result

\begin{tabular}{cccc}
\hline Parameter & $\mathrm{S} / \mathrm{SP}$ & $\mathrm{S} / \mathrm{P}$ & $\mathrm{S} / \mathrm{S}(\mathrm{CV})$ \\
\hline$V_{D C}[\mathrm{~V}]$ & 194 & 166 & 195 \\
$I_{D C}[\mathrm{~A}]$ & 4.36 & 5.02 & 4.53 \\
$V_{I N}[\mathrm{~V}]$ & 189 & 162 & 187 \\
$I_{I N}[\mathrm{~A}]$ & 5.32 & 6.03 & 9.77 \\
$P F_{I N}$ & 0.839 & 0.847 & 0.456 \\
$V_{\text {OUT }}[\mathrm{V}]$ & 85.7 & 161 & 92.0 \\
$I_{\text {OUT }}[\mathrm{A}]$ & 10.8 & 5.91 & 9.66 \\
$V_{L}[\mathrm{~V}]$ & 72.8 & 141 & 91.1 \\
$I_{L}[\mathrm{~A}]$ & 11.0 & 5.69 & 8.79 \\
$V_{I N} / V_{\text {OUT }}$ & 1.98 & 0.908 & 2.03 \\
\hline
\end{tabular}

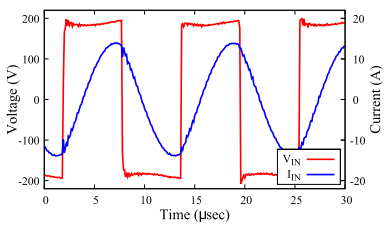

(a) Input

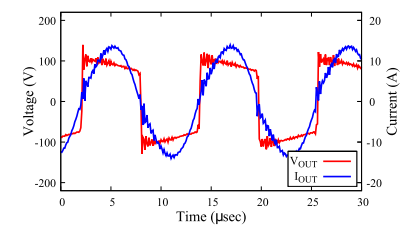

(b) Output
Fig. 6. Voltage/current waveforms of S/S (CV) system

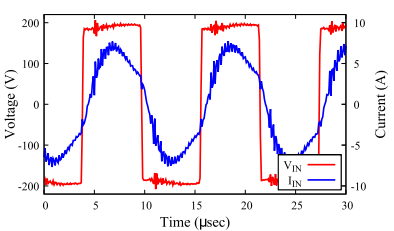

(a) Input

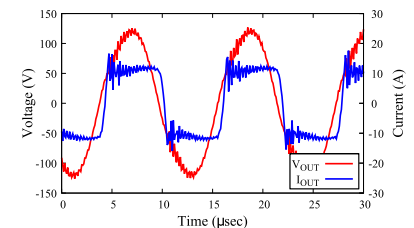

(b) Output
Fig. 7. Voltage/current waveforms of S/SP system

mean square (RMS) values of each parameter. The square wave parameters were calculated and listed as the square wave RMS values. As shown in Table $4, P F_{I N}$ of the $\mathrm{S} / \mathrm{S}$ (CV) system was 0.456 , whereas that of the S/SP system was 0.839 . This indicates that the power factor was improved by the parallel capacitor. The waveforms of the $\mathrm{I} / \mathrm{O}$ voltage and current of the S/S (CV) and S/SP system are shown in Figs. 6 and 7, respectively. From Figs. 6(a) and 7(a), it can be seen that $P F_{I N}$ was increased as well. In addition, the input current $I_{I N}$ had a delayed power factor with respect to $V_{I N}$, and it can be observed that zero voltage switching is realized ${ }^{(21)}$. The $\mathrm{I} / \mathrm{O}$ voltage ratio of the S/SP WPT circuit was 1.98 , which is consistent with the self-inductance ratio and almost the same as the turn ratio, validating the theory. Here, because $V_{I N}$ in each method and $V_{O U T}$ in the $\mathrm{S} / \mathrm{S}(\mathrm{CV})$ method were square waves, the I/O voltage ratio was calculated using 0.9 times the RMS value of the square wave, which is the RMS value of the fundamental wave.

Figure 8 shows the power transmitting efficiency of the three topologies. In comparison to the $\mathrm{S} / \mathrm{S}(\mathrm{CV})$ method, the $\mathrm{S} / \mathrm{SP}$ method showed higher values for both the transformer 


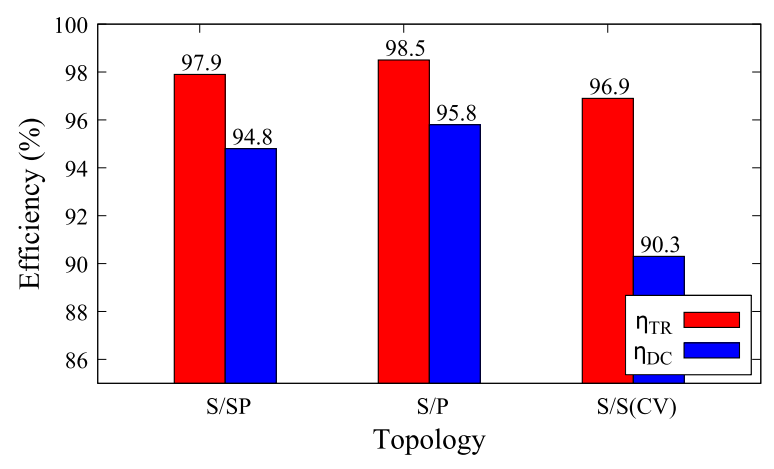

Fig. 8. Efficiency of three topologies

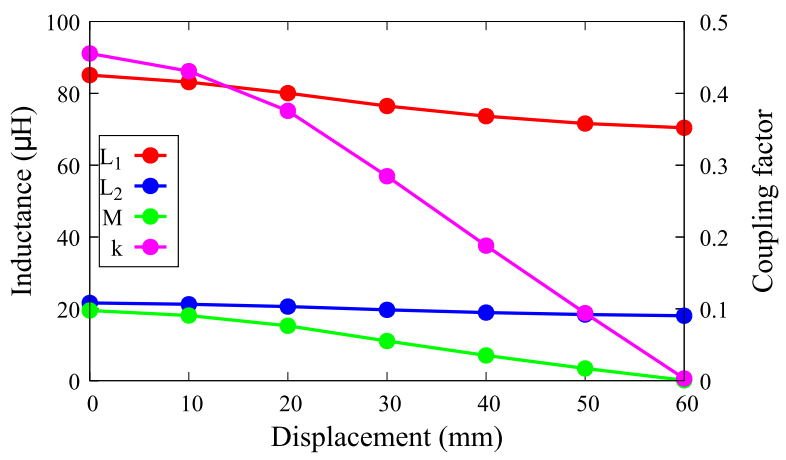

Fig. 9. Fluctuation of coil parameter

efficiency $\eta_{T R}$ and DC efficiency $\eta_{D C}$. In contrast, compared with the S/P method, the power transfer efficiency of the S/SP system was lower than that of the S/P system, but the difference was only $0.6 \%$ for $\eta_{T R}$ and $1.0 \%$ for $\eta_{D C}$. The result of $\eta_{T R}$ was in accordance with theory. It is considered that the difference in $\eta_{D C}$ was larger than the difference in $\eta_{T R}$ because $P F_{I N}$ of the $\mathrm{S} / \mathrm{P}$ system was higher than that of the S/SP system.

\subsection{Power Feeding in Secondary Misalignment}

Although our proposed method is intended for an application that is powered by placing it on a wireless charger, imagining an actual charging situation, since the device is roughly placed on the charger, it is essential to clarify power feeding characteristics during displacement. Therefore, we also evaluated the characteristics under the secondary coil misalignment through simulation and experiment. Figure 9 shows the fluctuation of each inductance and coupling coefficient when the secondary coil was displaced in the $X$ direction. The coupling coefficient $k$ decreased with the positional deviation and became almost zero at $60 \mathrm{~mm}$. The value of each inductance also decreased. The value of capacitors and load resistance were fixed at the value when there was no displacement.

Power supply simulation of the misalignment was performed using the circuit simulation software PSIM. In this simulation, $V_{D C}$ was adjusted to obtain $P_{L}$ of $200 \mathrm{~W}$ at the secondary alignment. $V_{D C}$ was kept constant in the case of positional deviation. Fluctuations of the DC input current $I_{D C}$ and $P_{L}$ during this simulation are shown in Fig. 10 and Fig. 11 respectively. $I_{D C}$ of both the $\mathrm{S} / \mathrm{P}$ and $\mathrm{S} / \mathrm{SP}$ systems increased according to the position shift. At the secondary alignment, the DC input current was $I_{D C}=2.81 \mathrm{~A}$ for the S/P system and was $I_{D C}=2.47 \mathrm{~A}$ for the S/SP system. At a position shift of $50 \mathrm{~mm}$, these were $I_{D C}=27.8 \mathrm{~A}$ for the $\mathrm{S} / \mathrm{P}$ system and $I_{D C}=$

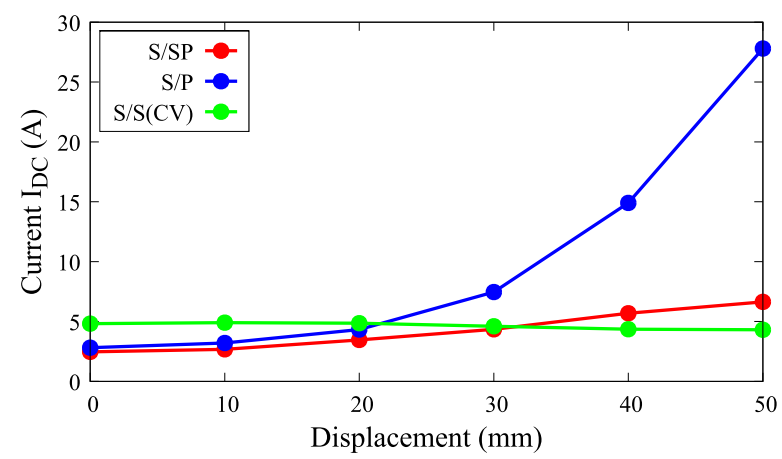

Fig. 10. Fluctuation of DC input current

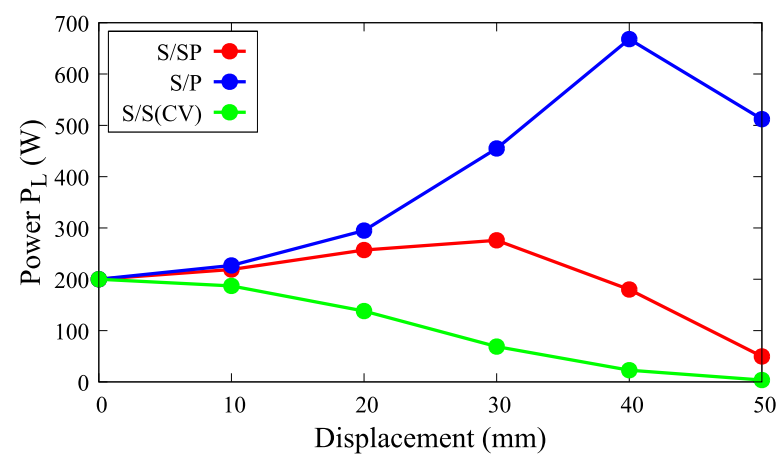

Fig. 11. Fluctuation of load power

6.64 A for the S/SP system. In the S/P system, the current at $50 \mathrm{~mm}$ was 9.90 times the current at the secondary coil alignment, while it was suppressed to 2.69 times in the S/SP system. In addition, the maximum load power was $P_{L}=668 \mathrm{~W}$ at $40 \mathrm{~mm}$ for the $\mathrm{S} / \mathrm{P}$ system and was $P_{L}=276 \mathrm{~W}$ at $30 \mathrm{~mm}$ for the S/SP. It can be said that the S/SP system, with a small increase in input current and load power, is superior than the $\mathrm{S} / \mathrm{P}$ system in terms of secondary displacement. On the other hand, in the $\mathrm{S} / \mathrm{S}(\mathrm{CV})$ system, $I_{D C}$ was almost constant but $P_{L}$ decreased according to the displacement. This was because the imaginary component of the input impedance increased and $P F_{I N}$ decreased in addition to the effect of the efficiency reduction.

Finally, we also conducted the experiment for secondary misalignment and compared the I/O voltage ratio and the power transmitting efficiency of the three topologies. In this experiment, $V_{D C}$ was varied to keep $P_{L}$ as always $200 \mathrm{~W}$ even in the case of displacement. This was done to prevent element breakdown owing to an increase in input current and unfairness due to differences in feeding power. The experimental results are summarized in Figs. 12, 13, 14, and 15. Although the compensation capacitances were fixed in the experiment, Fig. 12 showed that $C_{p 2}$ reduced the imaginary component of the input impedance even during displacement state. It is considered that this result was the reason why the fluctuation of the S/SP system with respect to the position shift was smaller than that of the S/S (CV) system. Figure 13 shows the $\mathrm{I} / \mathrm{O}$ voltage ratio during misalignment. The $\mathrm{S} / \mathrm{S}(\mathrm{CV})$ and $\mathrm{S} / \mathrm{SP}$ method have the same I/O relation during $\mathrm{CV}$ driving; however, the $\mathrm{S} / \mathrm{SP}$ system has a smaller voltage fluctuation with respect to the secondary displacement. In the S/SP method, the voltage ratio changed to smaller values due to the displacement, but the minimum value was 


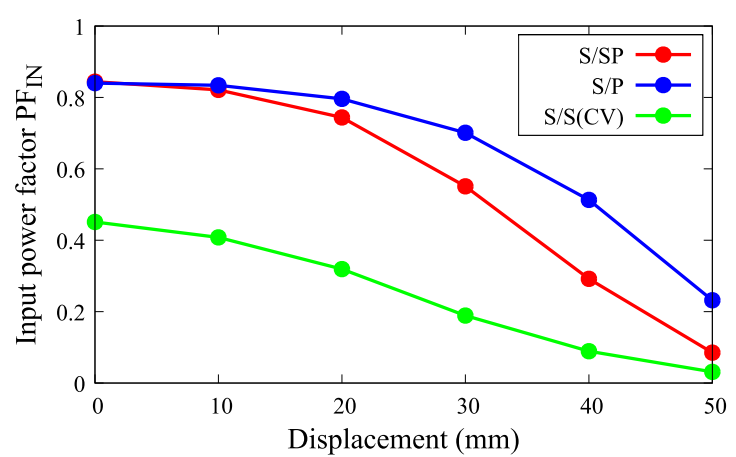

Fig. 12. Fluctuation of input power factor

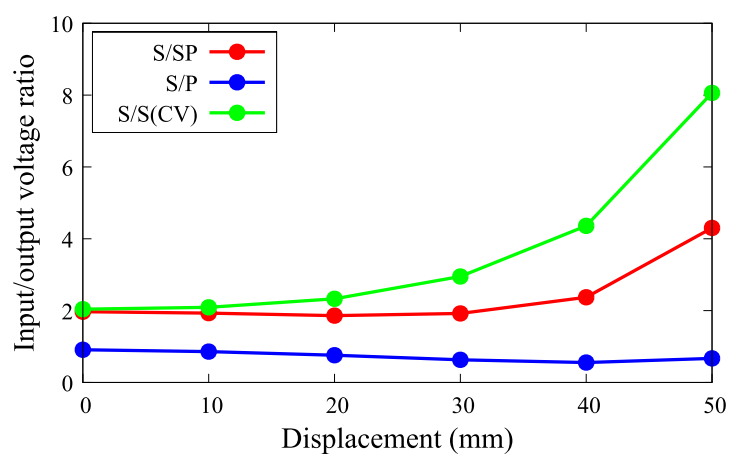

Fig. 13. Fluctuation of I/O voltage ratio

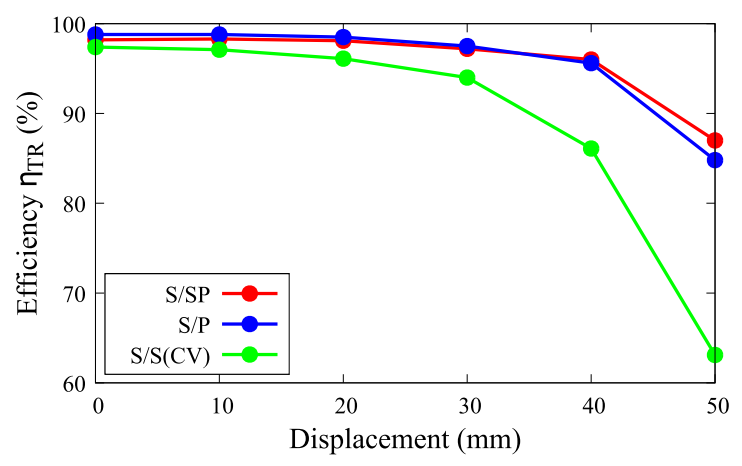

Fig. 14. Fluctuation of AC efficiency

1.86 at $20 \mathrm{~mm}$. Also, the ratio gradually increased as the displacement further advanced, reaching 2.37 at $40 \mathrm{~mm}$ and 4.30 at $50 \mathrm{~mm}$. The voltage difference between at $0 \mathrm{~mm}$ and at $20 \mathrm{~mm}$ was $8.58 \mathrm{~V}$, and the one between at $0 \mathrm{~mm}$ and at $40 \mathrm{~mm}$ was $22.5 \mathrm{~V}$. A general-purpose charging circuit usually has an operating voltage range of $20 \mathrm{~V}$ to $30 \mathrm{~V}$, so that there is a possibility that the S/SP method can be used even when the displacement is large.

Figure 14 shows that the fluctuation of transformer efficiency was smaller in the S/SP system than in the S/P system. Figure 15 displays that the DC efficiency was up to $2.40 \%$ higher in the $\mathrm{S} / \mathrm{P}$ system. However, the difference between the two efficiencies became smaller, and above $40 \mathrm{~mm}$, the $\mathrm{S} / \mathrm{SP}$ system was more efficient. This was because that the fluctuation of the optimum resistance in S/P method with respect to the displacement was bigger than that in S/SP method as shown in Fig. 16. These experimental results show that the S/SP system has better characteristics for secondary misalignment than the $\mathrm{S} / \mathrm{P}$ system.

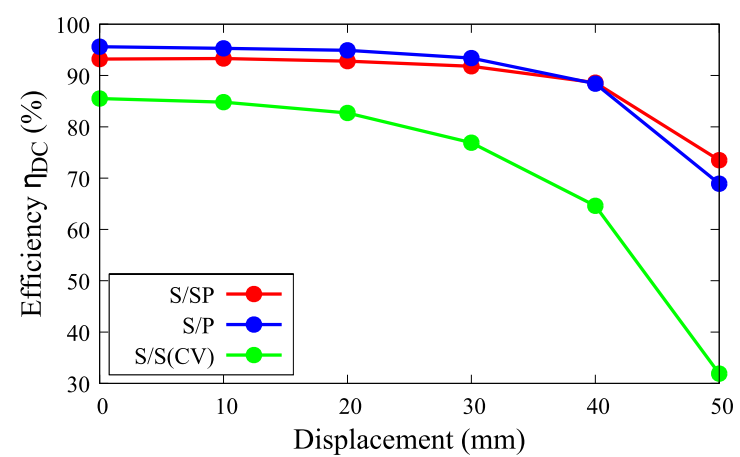

Fig. 15. Fluctuation of DC efficiency

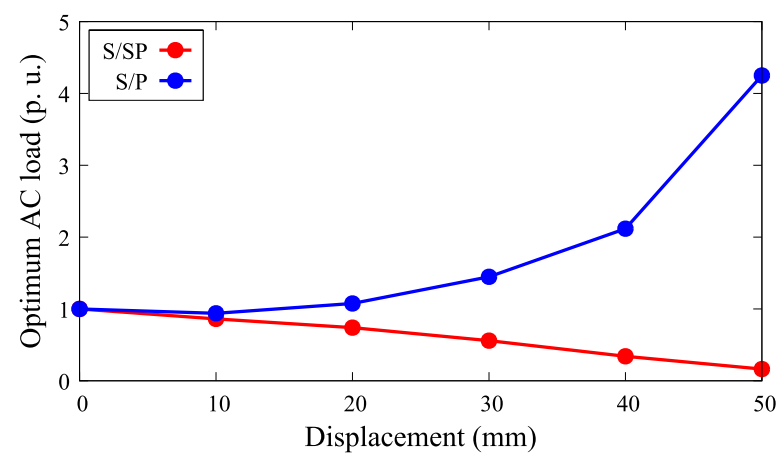

Fig. 16. Fluctuation of theoretical optimum resistance

\section{Conclusion}

As a solution to promote the introduction of WPT technology, we examined the WPT system that can use a generalpurpose DC power supply and charging converter. The proposed WPT circuit with the ideal transformer characteristics can easily step up/down the voltage by the WPT circuit section because the $\mathrm{I} / \mathrm{O}$ ratio of the WPT circuit is determined solely by the coils turn ratio, as in a general transformer. In the power feeding experiment for secondary alignment, the $\mathrm{I} / \mathrm{O}$ voltage ratio and self-inductance ratio coincided, and the value almost matched the turn ratio of the coils. In addition, compared with the S/P system with similar circuit configuration and characteristics as the S/SP system, the power transfer efficiency of the S/SP system was lower than that of the S/P system. However, the difference was only $0.6 \%$ for the $\mathrm{AC}$ efficiency and $1.0 \%$ for the DC efficiency, and it can be said that the S/SP method has the same power feeding capacity as the S/P method. Moreover, in simulation and experiment assuming secondary misalignment, the S/SP method showed little change with respect to displacement in terms of input current, load power and efficiency, and thus exhibited superior characteristics to the $\mathrm{S} / \mathrm{P}$ system.

In this study, the power feeding experiments and simulations were performed with solenoid type coils, but in the case of WPT where mounting in small products is taken into account circular coils, like a Qi standard ${ }^{(1)}$, have been extensively employed. Our future work investigates whether the $\mathrm{I} / \mathrm{O}$ ratio is obtained based on the turn ratio of the coils in the case of using circular coils as well. 


\section{References}

( 1 ) S.Y. Hui: "Planar wireless charging technology for portable electronic products and Qi", Proceedings of the IEEE, Vol.101, No.6 (2013)

( 2 ) X. Lu, P. Wang, D. Niyato, D.I. Kim, and Z. Han: "Wireless charging technologies: fundamentals, standards, and network applications", IEEE Communications Surveys and Tutorials, Vol.18, No.2 (2016)

( 3 ) G.A. Covic and J.T. Boys: "Inductive power transfer", Proceedings of the IEEE, Vol.101, No.6 (2013)

(4) W. Zhang and C.C. Mi: "Compensation topologies of high-power wireless power transfer systems", IEEE Trans. on Vehicular Technology, Vol.65, No.6 (2016)

( 5 ) X. Qu, Y. Jing, H. Han, S.-C. Wong, and C.K. Tse: "Higher order compensation for inductive-power-transfer converters with constant-voltage or constant-current output combatting transformer constraints", IEEE Trans. on Power Electronics, Vol.32, No.1 (2016)

( 6 ) T. Imura, H. Okabe, and Y. Hori: "Basic experimental study on helical antennas of wireless power transfer for electric vehicles by using magnetic resonant couplings", in Proc. 2009 IEEE Vehicle Power and Propulsion Conference (VPPC 09) (2009)

( 7 ) K. Nara, N. Madoiwa, and Y. Kaneko: "Wireless power transfer system whose input/output ratio is determined only by self-inductance", in Proc. 2019 IEEE MTT-S Wireless Power Transfer Conference (WPTC) (2019)

( 8 ) J. Hou, Q. Chen, K. Yan, X. Ren, S.-C. Wong, and C.K. Tse: "Analysis and control of S/SP compensation contactless resonant converter with constant voltage gain", in Proc. 2013 IEEE Energy Conversion Congress and Exposition (ECCE) (2013)

( 9 ) J. Hou, Q. Chen, X. Ren, X. Ruan, S.-C. Wong, and C.K. Tse: "Precise characteristics analysis of series/series-parallel compensated contactless resonant converter", IEEE Journal of Emerging and Selected Topics in Power Electronics, Vol.3, No.1 (2015)

(10) Y. Yao, X. Liu, Y. Wang, Y. Zhou, K. Lu, D. Xu, X. Liu, and H. Tian: "Analytical design of an S/SP compensated IPT system to minimize output voltage fluctuation under predetermined coupling coefficient range", in Proc. 2018 IEEE PELS Workshop on Emerging Technologies: Wireless Power Transfer (WoW) (2018)

(11) W. Zhang, S.-C. Wong, C.K. Tse, and Q. Chen: "Analysis and comparison of secondary series- and parallel-compensated inductive power transfer systems operating for optimal efficiency and load-independent voltage-transfer ratio", IEEE Trans. on Power Electronics, Vol.29, No.6 (2014)

(12) C. Kato, Y. Kaneko, K. Tsuda, T. Fujita, T. Matsumura, and T. Yasuda: "Investigation of Wireless Power Transfer System with Spaced Arranged Primary H-shaped Core Coils for Moving EVs", IECON 2015 - 41st Annual Conference of the IEEE Industrial Electronics Society (2015)

(13) Y. Moriwaki, T. Imura, and Y. Hori: "Basic study on reduction of reflected power using DC/DC converters in wireless power transfer system via magnetic resonant coupling”, in Proc. 2011 IEEE 33rd International Telecommunications Energy Conference (INTELEC) (2011)

(14) M. Kato, T. Imura, and Y. Hori: "Study on maximize efficiency by secondary side control using DC-DC converter in wireless power transfer via magnetic resonant coupling”, in Proc. 2013 World Electric Vehicle Symposium and Exhibition (EVS27) (2013)

(15) W.X. Zhong and S.Y.R. Hui: "Maximum Energy Efficiency Tracking for Wireless Power Transfer Systems", IEEE Transactions on Power Electronics, Vol.30, No.7 (2015)

(16) M. Fu, H. Yin, X. Zhu, and C. Ma: "Analysis and Tracking of Optimal Load in Wireless Power Transfer Systems", IEEE Transactions on Power Electronics, Vol.30, No.7 (2015)

(17) Q. Chen, S.C. Wong, C.K. Tse, and X. Ruan: "Analysis, design, and control of a transcutaneous power regulator for artificial hearts", IEEE Trans. on Biomedical Circuits and Systems, Vol.3, No.1 (2009)

(18) S.-Y. Cho, I.-O. Lee, S.C. Moon, G.-W. Moon, B.-C. Kim, and K.Y. Kim: "Series-series compensated wireless power transfer at two different resonant frequencies", in Proc. 2013 IEEE ECCE Asia Downunder (2013)
(19) R.L. Steigerwald: "A comparison of half-bridge resonant converter topologies", IEEE Transactions on Power Electronics, Vol.3, No.2 (1988)

(20) R. Bosshard, J.W. Kolar, J. Mülethaler, I. Stevanović, B. Wunsch, and F. Canales: "Modeling and $\eta$ - $\alpha$-Pareto optimization of inductive power transfer coils for electric vehicles", IEEE Journal of Emerging and Selected Topics in Power Electronics, Vol.3, No.1 (2015)

(21) S. Li, W. Li, J. Deng, T.D. Nguyen, and C.C. Mi: "A double-sided lcc compensation network and its tuning method for wireless power transfer", IEEE Trans. on Vehicular Technology, Vol.64, No.6 (2015)

Kenji Nara (Student Member) received the B.S. and M.S. in electrical and electronic systems engineering from Saitama University, Saitama, Japan, in 2018 and 2020, respectively. He has been working for Murata Manufacturing Co. Ltd. since 2020. His research interests are mainly on power electronics.

Naofumi Madoiwa (Student Member) received the B.S. degree in

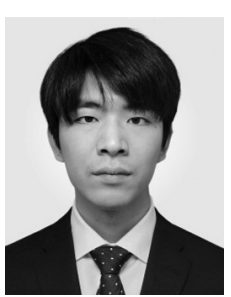
electrical and electronic systems engineering from Saitama University, Saitama, Japan, in 2019. He is currently working toward the M.S. degree in the department of electrical and electronic engineering with Tokyo Institute of Technology, Tokyo, Japan.

Keiri Maeshiro (Student Member) received the B.S. in electrical and electronic systems engineering from Saitama University, Saitama, Japan, in 2019. He is currently working toward the M.S. degree in department of mathematical and electronic information with Saitama University.

Yasuyoshi Kaneko (Member) received the B.S., M.S. and D.Eng. de-

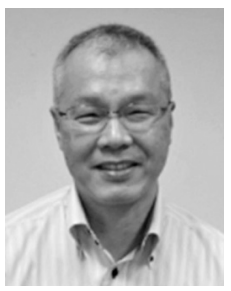
grees in electrical engineering from Saitama University, Saitama, Japan, in 1987, 1989 and 1994, respectively. In 1991, he joined the Department of Electrical Engineering, Saitama University as a Research Associate. He later became an Assistant Professor, an Associate Professor, and, in 2014, a Professor at the same university. His research fields include control of electrical equipment, intelligence information processing and control of industrial robots, etc. 